\title{
Risk Factors of Non-communicable Diseases and its Effective Surveillance with STEPwise Approach
}

\author{
M. Athar Ansari ${ }^{1}$, Ali Jafar Abedi ${ }^{2}$, M. Salman Shah ${ }^{3}$ \\ ${ }^{1}$ Professor, Department of Community Medicine, J.N. Medical College, A.M.U., Aligarh;'2Assistant Professor, Department of \\ Community Medicine, J.N. Medical College, A.M.U., Aligarh; ${ }^{3}$ Assistant Professor, Department of Community Medicine, J.N. \\ Medical College, A.M.U., Aligarh
}

Non-communicable diseases (NCDs) are responsible for almost $70 \%$ of all deaths worldwide. Almost $75 \%$ of all NCD deaths, and $82 \%$ of the 16 million people that died prematurely, or before reaching 70 years aged, occur in low and middle-income countries. The epidemic of NCDs including heart condition, stroke, cancer, diabetes, and chronic lung disease poses devastating health consequences for people, and threatens to overburden the health systems. In finances starved countries, the costs associated with NCDs make the management of these diseases a major development concern for the 21st century. ${ }^{[1]}$

\section{Risk Factors for NCDs}

Several risk factors have been identified in the high prevalence of NCDs, including smoking, obesity, and raised blood pressure, excessive intake of alcohol, each of which is associated with both NCDs and other negative health outcomes. ${ }^{[2]}$

\section{STEPwise approach}

To provide this information in developing countries, WHO has introduced the WHO STEPwise approach for surveillance of risk factors for non-communicable diseases in which 4 broad categories of NCDs namely, heart disease (coronary heart disease, stroke, and hypertension), diabetes, cancers and respiratory morbidities like COPD and asthma and associated risk factors have been considered. The WHO STEPwise approach to Surveillance (STEPS) of NCDs is based on sequential levels of surveillance of different aspects of noncommunicable diseases, allowing flexibility and integration at each step by maintaining standardized questionnaires and protocols to ensure comparability over time and across locations. ${ }^{[2]}$

It is based on two key premises: A collection of standardized data, and sufficient flexibility for use in a variety of country situations and settings.

\section{Methodology of STEPwise approach}

It provides a simple surveillance system based on 3 sequential steps for countries interested in developing appropriate NCD risk factor surveillance infrastructure.

- Step 1 includes a questionnaire-based survey for gathering information on key risk factors. In addition to socio-economic data, data on tobacco and alcohol use, some measure of nutritional status and physical inactivity are included as markers of current and future health status.

- Step 2 adds to Step 1 by the inclusion of simple physical measurements, such as blood pressure, height, weight, and waist circumference.

- Step 3 includes all Steps 1 and 2 and adds biochemical measures.

- All of the core items from Steps 1 and 2 can be readily assessed; inclusion of the expanded items adds little extra complexity. However, additional information in Step 3 is biochemical and requires access

Utilization of Data for Prevention of NCDs

Since the underlying risk factors of all the noncommunicable diseases are common,

CORRESPONDING AUTHOR: Dr M. Salman Shah, Department of Community Medicine, J.N. Medical College, A.M.U., Aligarh-202002

E Mail ID: salmanshah123@yahoo.com

ARTICLE CYCLE: Received: 03/02/2021; Revised: 07/02/2021; Accepted: 27/05/2021; Published:30/06/2021

CITATION: Ansari MA, Abedi AJ, Shah MS. Risk Factors of Non-communicable Diseases and its Effective Surveillance with STEPwise Approach.J Comp Health. 2021;9(1):46-47. 
identifying and modifying these risk factors have been recommended as a strategy for their prevention and control in various settings.

Prevention of NCDs could be a growing issue: the burden of NCDs falls principally on developing countries, where eighty two percent of premature deaths occur from these diseases. Grappling the chance factors can so not solely save lives; it will conjointly give an enormous boost for the economic development of states. ${ }^{[3]}$ This methodology helps to make a long-lasting closed-circuit surveillance system, and it will increase our trained workforce keeping in mind the various constraints of the states at the national level. Necessary data relating to NCDs are collected which can be utilized in the management of NCDs.

\section{REFERENCES}

1. Noncommunicable diseases. World Health Organization. Available: https://www.who.int/healthtopics/noncommunicable-diseases\#tab=tab_1 (Accessed on 10.07.2020)

2. STEPS: A framework for surveillance. The WHO STEPwise approach to Surveillance of noncommunicable diseases (STEPS) Noncommunicable Diseases and Mental Health, World Health Organization 2002.

3. Castaneda R, Sussman N, Westreich L, Levy R, O'Malley M. A review of the effects of moderate alcohol intake on the treatment of anxiety and mood disorders external icon. J Clin Psychiatry 1996;57(5):207-212. 